\title{
The Fundamental Plane of Globular Clusters
}

\author{
Dean E. McLaughlin \\ Space Telescope Science Institute, 3700 San Martin Drive, Baltimore, \\ $M D 21218$ USA
}

\begin{abstract}
The fundamental plane of globular clusters can be expressed as a particular scaling of cluster energy vs. mass: $E_{b} \sim M^{2}$. New calculations of energy and mass for a large sample of young clusters in the Magellanic Clouds may shed light on the origins of this scaling.
\end{abstract}

The fundamental plane of globular clusters (GCs) is typically defined in terms of velocity dispersion, radius, and surface brightness (Djorgovski 1995; Burstein et al. 1997). Comparisons in this parameter space show that GCs do not follow the same structural relations as early-type galaxies and bulges: while the radii of the latter depend on their masses, GC sizes do not; and while ellipticals follow the Faber-Jackson relation, $M \sim \sigma^{4}$, GCs instead obey $M \sim \sigma^{2}$ or so (e.g., Djorgovski \& Meylan 1994).

These scalings for GCs, $R \sim M^{0}$ and $\sigma \sim M^{0.5}$, are manifestly equivalent to a scaling between binding energy and total mass: $E_{b} \sim M^{2}$, as McLaughlin (2000) has confirmed by direct calculation. McLaughlin further shows how this energy-mass relation combines with a constant mass-to-light ratio in Galactic GCs to produce all other correlations between any set of GC observables, including the more traditional expressions of the fundamental plane. GCs in M31, M33, and NGC 5128 appear to follow the same mass-energy relation (Barmby et al. 2002; Larsen et al. 2002; Harris et al. 2002). On the other hand, the Faber-Jackson relation suggests $E_{b} \sim M^{1.5}$ for E galaxies and bulges.

The advantage of viewing the fundamental plane in terms of energy is that the processes of $\mathrm{GC}$ formation and dynamical evolution are ultimately ones of energetics. It is therefore significant that a scaling such as $E_{b} \sim M^{2}$ is much steeper than what is expected to have held in gaseous protoclusters $\left(E_{b} \sim M^{1.5}\right.$ again, quite generically; see, e.g., McLaughlin \& Pudritz 1996). It has been argued, though only heuristically, that the steeper $E_{b}(M)$ slope might have been imprinted during the birth of GCs, rather than over a Hubble time of dynamical evolution (McLaughlin 2000; Ashman \& Zepf 2001).

To check this idea empirically, we (D. E. McLaughlin \& R. P. van der Marel, in preparation) have calculated the masses and energies of Magellanic Cloud star clusters spanning a wide range of ages, from tens of Myr to 13 Gyr. Figure 1 shows our results. The values of $E_{b}$ and $M$ here follow from fitting structural models to the star-count data of Mackey \& Gilmore (2003a, b) and applying mass-to-light ratios derived from the population-synthesis code PEGASE (Fioc \& Rocca-Volmerange 1997). The apparent change of slope in $E_{b}$ vs. $M$ around $M \sim 1-2 \times 10^{5} M_{\odot}$ is a new feature, unrecognized in analysis of old and relatively massive GCs. Whether it is primarily an effect of age or of mass is not yet clear - though it is especially intriguing that it occurs at about the same 


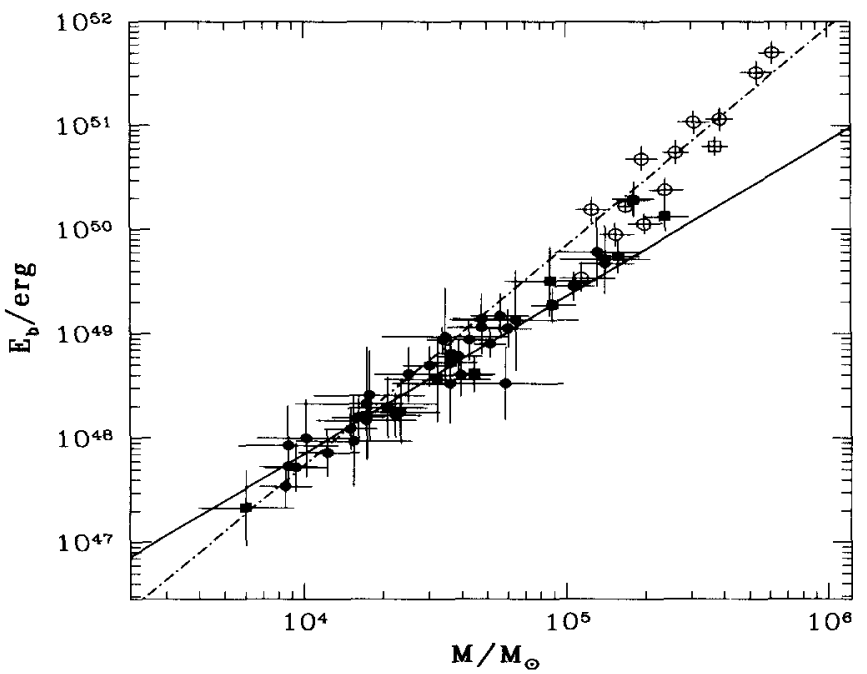

Figure 1. Binding energy vs. total mass for star clusters in the Magellanic Clouds. Filled circles: 36 young LMC clusters $\left(\tau \sim 10^{7}-10^{10} \mathrm{yr}\right)$. Open circles: 12 old LMC globulars. Filled squares: 9 young SMC clusters. Open square: one old SMC globular. Solid line: $E_{b} \sim M^{1.5}$, as expected for gaseous protoclusters. Broken line: $E_{b} \sim M^{2.1}$, roughly as found for old GCs in the Milky Way, M31, M33, and NGC 5128.

mass scale which marks the turnover of the globular cluster luminosity function. Although it must be noted that the result depends critically on the cluster massto-light ratios predicted by the PEGASE code, understanding it may eventually provide important new insight into the overall properties of GCs as a class.

Acknowledgments. I am grateful to the American Astronomical Society for support in the form of an International Travel Grant.

\section{References}

Ashman, K. M., \& Zepf, S. E. 2001, AJ, 122, 1888

Barmby, P., Holland, S., \& Huchra, J. P. 2002, AJ, 123, 1937

Burstein, D., Bender, R., Faber, S., \& Nolthenius, R. 1997, ApJ, 114, 1365

Djorgovski, S. 1995, ApJ, 438, L29

Djorgovski, S., \& Meylan, G. 1994, AJ, 108, 1292

Harris, W. E., Harris, G. L. H., Holland, S. T., \& McLaughlin, D. E. 2002, AJ, 124, 1435

Fioc, X., \& Rocca-Volmerange, Y. 1997, A\&A, 326, 950

Larsen, S. S., Brodie, J. P., Sarajedini, A., \& Huchra, J. P. 2002, AJ, 124, 2615

Mackey, A. D., \& Gilmore, G. 2003a, MNRAS, 338, 85

Mackey, A. D., \& Gilmore, G. 2003b, MNRAS, 338, 120

McLaughlin, D. E. 2000, ApJ, 539, 618

McLaughlin, D. E., \& Pudritz, R. E. 1996, ApJ, 469, 194 